EPJ Web of Conferences 43, 03012 (2013)

DOI: $10.1051 /$ epjconf/20134303012

(C) Owned by the authors, published by EDP Sciences, 2013

\title{
Internal rotation of red giants by asteroseismology
}

\author{
M.P. Di Mauro ${ }^{a}$, D. Cardini ${ }^{1}$, R. Ventura², D. Stello ${ }^{3}$, P.G. Beck ${ }^{4}$, \\ G. Davies ${ }^{5}$, Y. Elsworth ${ }^{6}$, R.A. García ${ }^{5}$, S. Hekker ${ }^{7}$, B. Mosser ${ }^{8}$, \\ J. Christensen-Dalsgaard ${ }^{9}$, S. Bloemen ${ }^{4}$, G. Catanzaro ${ }^{2}$, \\ K. De Smedt ${ }^{4}$ and A. Tkachenko ${ }^{4}$
}

${ }^{1}$ INAF-IAPS Roma, Italy

${ }^{2}$ INAF-Osservatorio Astrofisico di Catania, Italy

${ }^{3}$ Sydney Institute for Astronomy, School of Physics, University of Sydney, Australia

${ }^{4}$ Instituut voor Sterrenkunde, Katholieke Universiteit Leuven, Belgium

${ }^{5}$ AIM, CEAIDSM-CNRS-Université Paris Diderot, IRFU/Sap., Centre de Saclay, France

${ }^{6}$ School of Physics and Astronomy, University of Birmingam, UK

${ }^{7}$ Astronomical Institute Anton Pannekoek, University of Amsterdam, The Netherlands

${ }^{8}$ LESLIA, CNRS, Université Pierre et Marie Curie, Université Denis Diderot, Observatoire de Paris, Meudon Cedex, France

${ }^{9}$ Department of Physics and Astronomy, Aarhus University, Denmark

\begin{abstract}
We present an asteroseismic approach to study the dynamics of the stellar interior in red giant stars by asteroseismic inversion of the splittings induced by the stellar rotation on the oscillation frequencies. We show preliminary results obtained for the red giant KIC4448777 observed by the space mission Kepler.
\end{abstract}

\section{ASTEROSEISMIC DATA}

The red giant KIC4448777 has been continuously observed by the Kepler satellite for 670 days in long cadence mode (integration time of $30 \mathrm{~min}$ ). The Fourier analysis of the long time series has shown a clear power excess between $180-260 \mu \mathrm{Hz}$ (Fig. 1) and allowed us to identify 58 individual modes characterized by a mean large frequency separation $\Delta v=16.96 \pm 0.03 \mu \mathrm{Hz}$, a true period spacing $\Delta \mathrm{P}=90 \pm 3 \mathrm{~s}$ [1] and a frequency of the maximum amplitude of the smoothed excess power of $v_{\max }=219.75 \pm 1.23 \mu \mathrm{Hz}$. The observed modes are $l=0$ pure acoustic modes, and $l=1, l=2$ and $l=3$ modes with mixed gravity-pressure character.

\section{ROTATIONAL SPLITTINGS}

Rotation breaks the spherical symmetry of the stellar structure and splits the frequencies of normal modes in $(2 l+1)$ components. Figure 1 shows the spectrum of KIC4448777 which is characterized by the presence of 15 rotational splittings for $l=1$. As it has been noticed by [2], the observed rotational splittings are not constant for consecutive dipole modes (see lower panel of Fig. 1): they are larger for modes with larger gravity component which probes better the core. This indicates that the core of this star is rotating faster than the upper layers. In order to quantify the internal rotation it is possible to invert

\footnotetext{
ae-mail: maria.dimauro@inaf .it
}

This is an Open Access article distributed under the terms of the Creative Commons Attribution License 2.0, which permits unrestricted use, distribution, and reproduction in any medium, provided the original work is properly cited. 
Figure 1. The upper panel shows the oscillation spectrum of KIC4448777. The harmonic degree of the observed modes $(l=0,1,2,3)$ are indicated. Multiplets due to rotation are visible for $l=1$. The lower panel shows the values of the observed rotational splitting for individual $l=1$ modes.

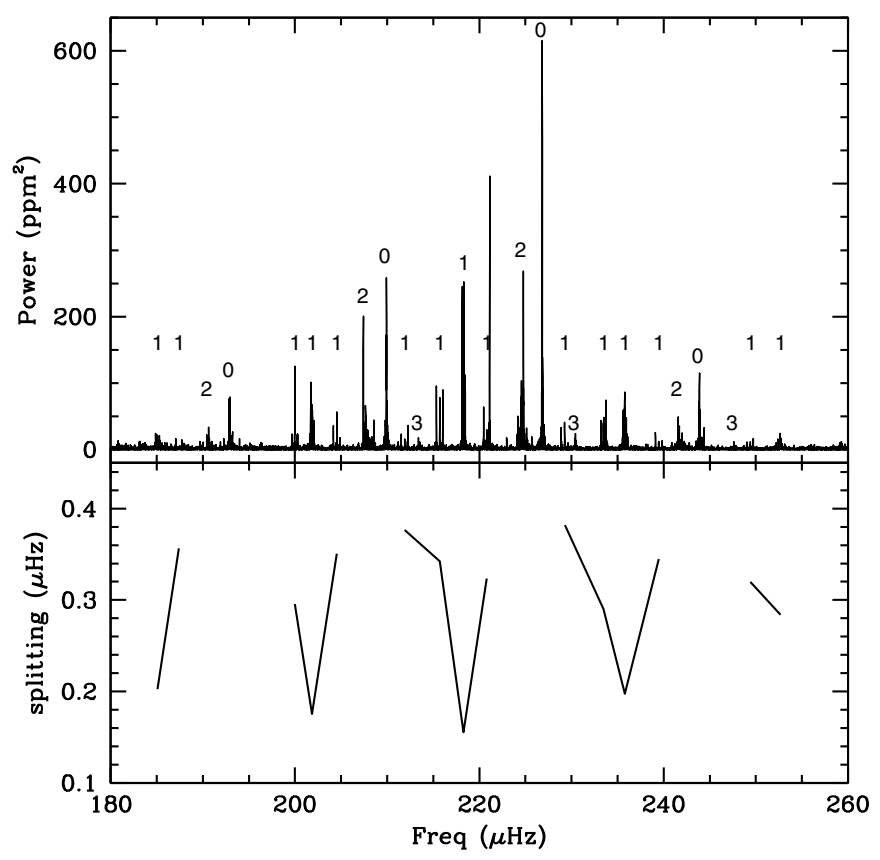

Table 1. Atmospheric parameters.

\begin{tabular}{|l|c|}
\hline$M_{v}$ & 11.56 \\
\hline$T_{\text {eff }}(\mathrm{K})$ & $4750 \pm 250$ \\
\hline $\log g(\mathrm{dex})$ & $3.5 \pm 0.5$ \\
\hline$[\mathrm{Fe} / \mathrm{H}]$ & $0.23 \pm 0.12$ \\
\hline$v \sin \mathrm{i}(\mathrm{km} / \mathrm{s})$ & $<5$ \\
\hline
\end{tabular}

Table 2. Parameters of the best fitting models.

\begin{tabular}{|l|c|c|}
\hline & Model 1 & Model 2 \\
\hline$M / M_{\odot}$ & 1.02 & 1.13 \\
\hline$T_{\text {eff }}(\mathrm{K})$ & 4800 & 4735 \\
\hline $\log \mathrm{g}($ dex $)$ & 3.26 & 3.27 \\
\hline$R / R_{\odot}$ & 3.94 & 4.08 \\
\hline$L / L_{\odot}$ & 7.39 & 7.50 \\
\hline$(Z / X)_{i}$ & 0.022 & 0.032 \\
\hline
\end{tabular}

the following equation, obtained by applying a standard perturbation theory to the eigenfrequencies, relating the splittings $\delta v_{n, l}$ to the internal rotation $\Omega(\mathrm{r})$ :

$$
\delta v_{n, l}=\int_{0}^{R} K_{n, l}(r) \frac{\Omega(r)}{2 \pi} d r
$$

where $K_{n, l}(r)$ are the mode kernel functions calculated on the unperturbed eigenfunctions for the modes $(n, l)$ of the 'best' model of the star and $R$ is the photospheric stellar radius.

\section{RESULTS AND CONCLUSION}

The theoretical structure models which better reproduce the identified pulsational frequencies have been calculated with the ASTEC evolution code [3] assuming the basic atmospheric parameters given in the 
Ageing Low Mass Stars: From Red Giants to White Dwarfs

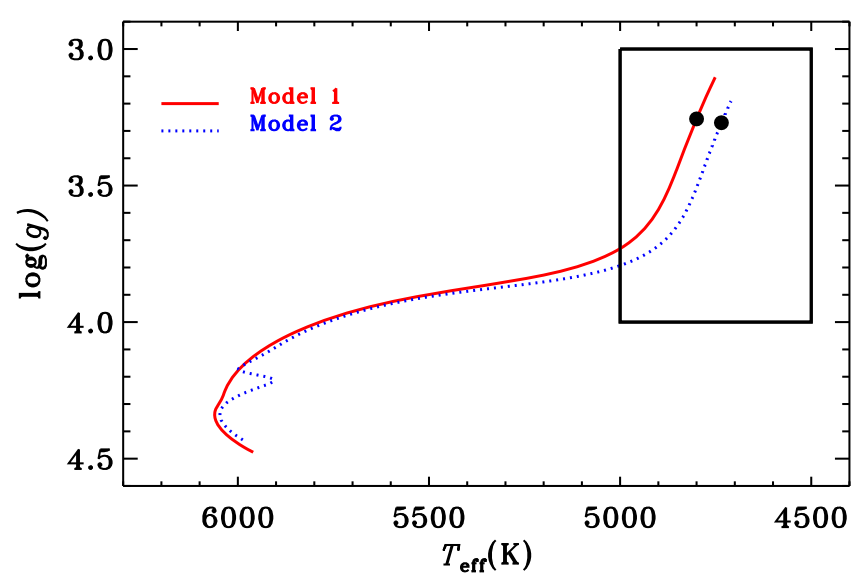

Figure 2. Evolutionary tracks plotted in a $\mathrm{H}-\mathrm{R}$ diagram. Black dots indicate two models which best reproduce the observations.
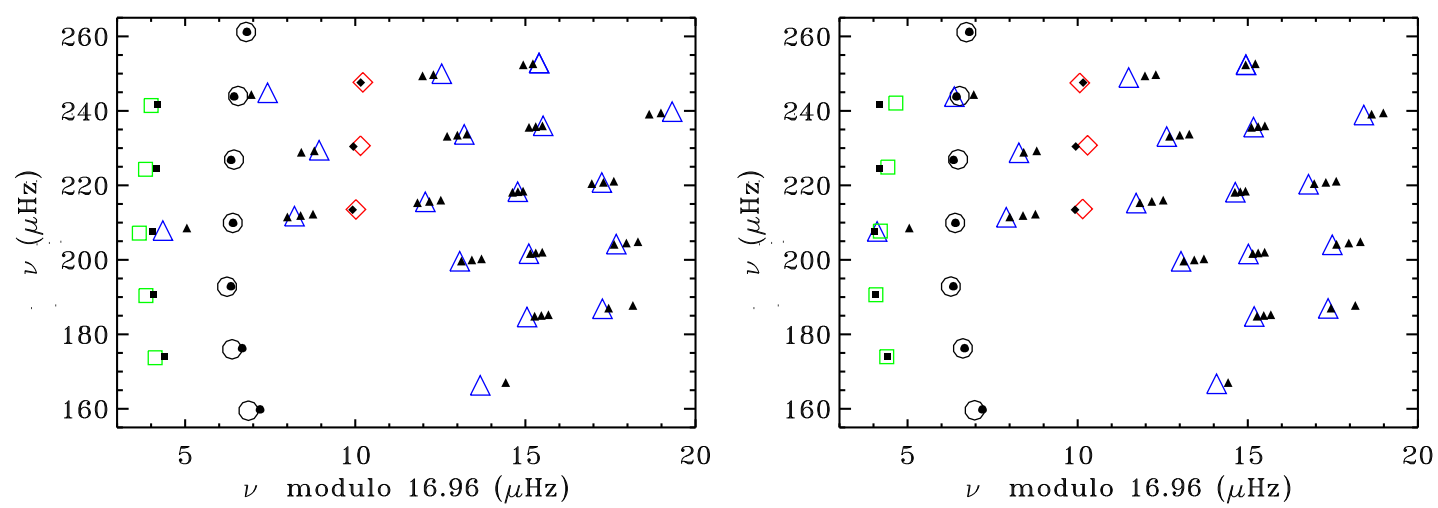

Figure 3. Echelle diagrams for Model 1 (on the left) and Model 2 (on the right) of Table 2. The filled symbols show the observed frequencies. The open symbols show the computed frequencies. Circles are used for modes with $l=0$, triangles for $l=1$, squares for $l=2$, diamonds for $l=3$.

Table 1. These parameters have been obtained by the analysis of the spectra taken with the Hermes spectograph [4] mounted on the $1.2 \mathrm{~m}$ Mercator telescope located in the Canary Islands. Adiabatic oscillation frequencies have been calculated with the ADIPLS code [5] and corrected for the surface effect by using the relation proposed by [6]. The mass, the effective temperature, the gravity, the surface radius, the luminosity and the initial metallicity of the two models which best fit the observations are given in Table 2. These values indicate that this star is in the hydrogen-shell burning phase (see Fig. 2), as predictable from the period spacing.

Figure 3 shows the echelle diagram obtained for one of the best fitting models. In a close future we plant to invert Eq. (1) by using both the OLA (Optimally localized Averaging) and the SOLA (Subtractive Optimally Localized Averaging) techniques which were successfully applied to the Sun (see e.g. [7]; [8]). These techniques allow to estimate a localized weighted average of $\Omega(r)$ making attempts to fit the averaging kernels to a function of fixed width and centered at a chosen value of the radius. Results will thus give us quantitative information on the differential rotation of the interior of the examined star. 
EPJ Web of Conferences

\section{References}

[1] T.R. Bedding, B. Mosser, D. Huber et al. Nature 471, (2011) 608-611

[2] P.G. Beck, J. Montalban, T. Kallinger, J. De Ridder et al., Nature 481, (2012) 55-57

[3] J. Christensen-Dalsgaard, ApSS 316, (2008) 13-24

[4] G. Raskin, H. van Winckel, H. Hensberge, et al., A\&A 526, (2011) 69-81

[5] J. Christensen-Dalsgaard, ApSS 316, (2008) 113-120

[6] H. Kjeldsen, T.R. Bedding, J. Christensen-Dalsgaard, ApJ 683, (2008) L175-L178

[7] L. Paternó, S. Sofia, M.P. Di Mauro, A\&A 314, (1996) 940-946

[8] M.P. Di Mauro and W.A. Dziembowski, Mem. Sait 69, (1998) 559-562 